\title{
Mejorar la competencia social y ciudadana: innovación desde educación físicay tutoría The improvement of the social and citizen competence: innovation from physical education and tutoring Núria Monzonís Martínez, Marta Capllonch Bujosa Universidad de Barcelona (España)
}

Resumen. Este artículo presenta una intervención basada en un proyecto de investigación-acción llevado a cabo en un centro de Educación Primaria durante el curso 2012-13. Su objetivo era la adquisición de las capacidades de la competencia social y ciudadana del alumnado con la intención de prevenir y resolver positivamente los conflictos y, mejorar con ello el clima de convivencia en el aula. Este proyecto se llevó a cabo implicando las áreas de Educación Física y las sesiones que el centro destinaba específicamente a la tutoría. Tras una fase de diagnóstico del clima de convivencia en las clases de Educación Física del tercer ciclo de Primaria, se priorizaron las habilidades a trabajar con el alumnado y se diseñaron las acciones a desarrollar. Esta organización permitió introducir y reflexionar sobre las habilidades sociales en las sesiones de tutoría y facilitó su puesta práctica en situaciones reales en las sesiones de Educación Física. Una vez valorados los resultados, se puede concluir que la experiencia aportó una considerable disminución del número de conflictos, tanto en el aula ordinaria como en la Educación Física.

Palabras clave. Educación Física, competencia social y ciudadana, resolución de conflictos, Investigación-acción, convivencia, tutoría, aprendizaje cooperativo.

Abstract. This paper addresses intervention based of an action-research project undertaken by a Primary Education center during the 2012-13 school year. The objective was to acquire skills related to the student's social and citizen competence with the intention of preventing and positively resolving conflicts, and thereby improving the classroom community and environment. This project was carried out using areas of physical education and the specific sessions that canter dedicated to tutoring. After the initial diagnostic period of the classroom community during physical education classes from the 5th and 6th grade of primary school, the priority became working with the skills of the students to design an action plan. This structure allowed for the introduction and reflection upon social skills during these sessions in the tutoring class as well as the application of these skills to real life situations in physical education. Once the results were evaluated, it was concluded that there was a significant decrease in the amount of conflicts in the classroom as well as physical education.

Key words. Physical Education, social and citizen competence, conflict resolution, action-research, classroom community, tutoring classes, collaborative learning.

\section{Introducción}

Actualmente vivimos en una sociedad cada vez es más globalizada, plural y multicultural, que nos ofrece la oportunidad de convivir y relacionarnos con personas muy diferentes (Bolívar \& Moya, 2007). Sin embargo, algunos consideran que en esta sociedad escasea la participación crítica y comprometida por el cambio social, y que a la vez esta influenciada por valores mediáticos como: individualismo, éxito, poder, competitividad o agresividad (Roberts, Yeomans \& FerroAlmeida, 2007). Y es que en efecto, estamos rodeados de modelos y referentes agresivos que tienen una seria repercusión en la infancia. Muchos niños experimentan, aprenden e imitan comportamientos violentos como modo de comunicarse y establecer relaciones con los demás (Felip, 2013). Por lo tanto, podríamos decir que vivimos en una sociedad en contraposición de valores que lleva a una socialización desequilibrada de los alumnos que afecta al transcurso de la vida escolar (Gázquez, Pérez \& Carrión, 2011; Moreno, 2012; Moreno, Estévez, Murgui \& Musitu; 2009).

La escuela no queda aislada de esta dinámica social. El nivel de conflictividad en las aulas ha incrementado y consecuentemente ha generado una creciente preocupación por la convivencia escolar (Cerezo, 2007). Por ello, será importante que la escuela no sólo instruya, sino que debe ir más allá para frenar el incremento de la conflictividad que se da en su contexto. Desde esta perspectiva, las aulas pueden convertirse en un entorno idóneo para la reconstrucción de la sociedad, el remedio de posibles injusticias y la cimentación de los principios de ciudadanía activa (Bolívar \& Moya, 2007).

Así pues, una de las funciones de la Institución Escolar deberá ser preparar para la vida apoyando el pleno desarrollo de cada individuo, procurando que adquieran actitudes y valores necesarios para un desarrollo personal y social que les permitan adaptarse a las necesidades del entorno y así desarrollar una

Fecha recepción: 12-10-14- Fecha envío revisores: 12-10-14- Fecha de aceptación: 25-03-15 Núria Monzonís

nmonzoni@xtec.cat buena competencia social y ciudadana (CSC, de aquí en adelante) (González, Catalán \& Lara, 2011).

Estas ideas siguen los planteamientos educativos que parten del desarrollo de las competencias básicas (CCBB, de aquí en adelante), ya que se entiende que tienen que ver con la capacidad de los estudiantes de extrapolar lo que han aprendido para aplicar sus conocimientos en nuevas circunstancias, así como conectar su aprendizaje a lo largo de la vida con regularidad (OCDE, 2007). Está conexión entre competencias y vivencias contribuirá al despliegue de una trayectoria personal exitosa y al buen funcionamiento de la sociedad, porque son importantes para las esferas de la vida del individuo y para la sociedad (Escamilla, 2008). Bolívar y Moya (2007) consideran que para conseguir este éxito y ser ciudadano activo se requieren unas competencias (comprensión lectora, matemática, científica y nuevas alfabetizaciones) que permitan al individuo integrarse en la vida social y laboral; pero por otro lado, algunos autores (Doncel \& Leena, 2011; Marco Stiefel, 2008) llegan a considerar que las destrezas y habilidades de la CSC son los pilares de la relación educativa y del resto de las CCBB; ya que sin una buena convivencia y un buen manejo de las emociones se hace más difícil la tarea de aprender. Conocerse, conducirse, conocer a los demás, manejar las habilidades sociales, autorrealizarse y convivir en las sociedades actuales son metas a consolidar dentro del ámbito escolar para favorecer el desarrollo, la madurez personal y la adaptación social y ciudadana de nuestro alumnado (Doncel \& Leena, 2011). Apoyando esta idea, Moreno (2012), afirma que para saber vivir sobre todo hay que saber convivir, lo que hará de la CSC un elemento imprescindible para la educación del siglo XXI. Y es que la CSC supone:

Comprender la realidad social en que se vive, afrontar la convivencia y los conflictos empleando el juicio ético basado en los valores y prácticas democráticas, y ejercer la ciudadanía actuando con criterio propio, contribuyendo a la construcción de la paz y la democracia, y manteniendo una actitud constructiva, solidaria y responsable ante el cumplimiento de los derechos y obligaciones cívicas. (Real Decreto 1631/2006, p. 689).

Lograrlo dependerá de que el alumno integre el conocimiento y comprensión de los valores en los que se basa la sociedad 
democrática, a través de la reflexión crítica de los conceptos de: democracia, libertad, solidaridad, corresponsabilidad, participación, ciudadanía y atención a los derechos y deberes de las declaraciones internacionales, la Constitución española y las legislaciones autonómicas (González et al., 2011). Además de estos conceptos, el alumno necesitará habilidades que le permitan participar, tomar decisiones y elegir cómo comportarse en diferentes situaciones, responsabilizándose de las elecciones tomadas a la vez que «siendo consciente de sus creencias, valores, sentimientos, actitudes y habilidades sociales para autorregular su conducta social y el ejercicio de la convivencia cívica» (Doncel \& Leena, 2011, p. 47). En cuanto a las actitudes que desarrolla esta competencia se encuentran la tolerancia, la cooperación, la escucha activa, el diálogo, la solidaridad, la empatía, el compromiso en la defensa de los derechos humanos y los valores necesarios para la convivencia (González et al., 2011).

Dado el efecto negativo que la sociedad puede ejercer sobre el desarrollo social del alumnado, la escuela debería contrarrestar estas carencias empezando el trabajo de la CSC en edades tempranas. Así mismo, debería fomentar la prevención y resolución de conflictos (RC, de aquí en adelante), la convivencia pacífica, y la reducción de conductas violentas (Ortega, Del Rey, Córdova \& Romera, 2008). Por lo tanto, enseñar estrategias y procedimientos para mejorar la convivencia en la escuela será una forma de asegurar que todo el alumnado adquiera habilidades sociales y de aprovechar las potencialidades del vivir en sociedad (Marina \& Bernabeu, 2007).

Desde esta perspectiva, no podemos olvidar que la Educación Física (EF, de aquí en adelante) promoverá sus competencias específicas y a la vez contribuirá en la consecución de las CCBB porque busca el desarrollo de la persona en su globalidad implicando aspectos motrices, cognitivos, efectivos y sociales (Blázquez, 2009). Además como la EF se plantea como un área principalmente social y vivencial, donde la interacción es casi implícita, tendrá un gran potencial pedagógico en el campo de la CSC (Hernández \& Rodríguez, 2011; Lleixà, 2007; Vaca, 2008). Podemos decir que la EF ayuda a aprender a convivir, desde la elaboración y aceptación de las reglas, desde el respeto a la autonomía personal, la participación, la cooperación, la desinhibición, el liderazgo, la resolución de conflictos, la empatía y la valoración de la diversidad (González et al., 2011).

Moreno (2012) también defiende que la EF favorece la consecución de la CSC porque enseña a convivir y a adquirir habilidades sociales facilitando la relación, la autonomía, la integración, el respeto, la cooperación y la solidaridad. La comprensión crítica de la realidad exige experiencias que la EF puede aportar con un buen enfoque metodológico y un buen planteamiento de sus contenidos. Puesto que en el desarrollo normal de las sesiones el alumnado esta en constante relación, se requiere de habilidades para conocerse y valorarse, expresar y escuchar, ponerse en el lugar del otro, decidir, valorar intereses propios y del equipo, entender la dialéctica entre diferencias personales e igualdad en los derechos colectivos (Vaca, 2008). Esto hace que las actividades físicas sean un medio integrador (Norlena \& Mohd, 2010; Osman, Dilsad \& Miiberra, 2010) que desarrolla elementos clave de la CSC como el respeto, la aceptación de las posibilidades propias y de los demás, el desarrollo de la cooperación, la igualdad y el trabajo en equipo.

Por lo tanto, una buena programación de las actividades permitirá aprender de la diversidad, apreciar la ciudadanía global y poner en práctica las habilidades sociales, así como aceptar los códigos de conducta y reglamentos; y desarrollar roles de liderazgo, el esfuerzo compartido y el valor del grupo (Gómez \& Prat, 2009; Moreno, 2012).

Diferentes autores del panorama nacional e internacional han aprovechando el potencial del carácter interactivo de la EF para tratar diferentes aspectos de la CSC como: los valores y las actitudes (Sánchez-Oliva, Leo, Amado, González-Ponce y López, 2012; Venero, 2007), las habilidades sociales y la responsabilidad (Hellison, 1995; Moreno-Murcia, Gónzalez-Cutre y Sicilia, 2007; Norlena y Mohd, 2010; Pascual, Escartí, Llopis, Marín y Wright, 2011), la cooperación (Casey, Dyson y Campbell, 2009; Osman et al., 2010; (Fernández Rio, 2003; Fernández-Río \& Velázquez, 2005; Velázquez Callado, 2004, 2008, 2013) o la RC (Garrett \& Wrench, 2011; Martín \& Ríos, 2014; Ruíz Omeñaca, 2008).

Una vez situados en este marco de referencia, el objetivo del presente trabajo es mostrar una propuesta de innovación centrada en el desarrollo de la CSC del alumnado a través del trabajo interdisciplinar entre las áreas de tutoría y de EF. Esta interdisciplinariedad buscó que desde la tutoría se presentasen las diferentes dimensiones de la CSC, para después aplicarlas en situaciones reales dadas en EF. Cabe destacar que toda la intervención se enmarcó en una investigación-acción (I-A, de aquí en adelante), realizada en los cursos 2011-12 y 2012-13, que buscaba contrarrestar las dinámicas negativas que se daban en las interrelaciones del alumnado y así mejorar el clima de convivencia de un centro escolar de Educación Primaria.

\section{Material y métodos}

\section{Contextualización}

Este proyecto de innovación configurado a través de una IA se llevó a cabo en un Instituto-Escuela público de Barcelona, situado en el distrito de Nou Barris. El nivel socio económico de las familias que forman parte de la comunidad educativa del centro puede considerarse bajo o muy bajo. Las familias son de origen sudamericano en un 50\%, y un $20 \%$ es de etnia gitana. El resto son españoles o de una amalgama de culturas de diferentes países. El centro se constituyó en el curso 2011-12 tras la fusión de dos centros de Educación Primaria y uno de Educación Secundaria.

Tras el primer curso de adaptación, el profesorado del centro ya sentía que en el día a día existían serios problemas de convivencia entre el alumnado y se daban graves faltas de respeto hacia los adultos. Puesto que entre los estudiantes del tercer ciclo de educación primaria los niveles de conflictividad eran los más elevados del centro, se decidió intervenir específicamente para mejorar la situación. La idea inicial fue aprovechar las ventajas de la EF para trabajar aspectos de la CSC y mejorar la prevención y RC; pero posteriormente se decidió incrementar los tiempos de reflexión y llevar a cabo un proyecto de innovación conectando EF con la sesión semanal de tutoría, que estaba instaurada en el centro desde primero de primaria hasta cuarto de secundaria.

\section{Participantes}

La intervención se llevó a cabo en un grupo-clase de quinto de primaria. Para la selección de la muestra se siguió el criterio de accesibilidad, puesto que era el grupo en el que coincidía que el profesor de Educación Física y el responsable de la tutoría eran la misma persona, lo cual permitía una acción conjunta desde los dos ámbitos.

\section{La investigación-acción}

La I-A permite dotar de rigor científico a la investigación educativa (Latorre, 2003) ofreciendo un soporte sistemático al diseño, desarrollo y evaluación de una acción, que en este caso buscaba la mejora de la convivencia. En este punto, debemos destacar la reflexión como elemento que impulsa la I-A para comprender el contexto en el que se actúa, transformar las prácticas educativas y desarrollar nuevos conocimientos (Hine \& Lavery, 2014). Dadas estas características, la I-A se ajustó a las 


\begin{tabular}{|c|c|c|c|}
\hline \multicolumn{4}{|r|}{ Temporizarín } \\
\hline $\mathrm{I}-\mathrm{A}$ & Acciones & Herramientas & Temporización \\
\hline \multirow{5}{*}{ Planificación } & Identificación del problema & & Curso 2010-11 \\
\hline & \multirow[b]{2}{*}{ Diagnóstico del estado inicial } & Cuestionario, para el alumnado & Abril 2011 \\
\hline & & $\begin{array}{l}\text { Observación sistemática de las } \\
\text { sesiones de EF }\end{array}$ & 3r trim. 2011-12 \\
\hline & Formación del grupo de trabajo & $\begin{array}{c}\text { Encuesta profesorado: } \\
\text { priorización habilidades sociales }\end{array}$ & $2^{\circ}$ trim. 2011-12 \\
\hline & Diseño de la intervención & Comisión de docentes & 3r trim. 2011-12 \\
\hline Acción & Aplicación del programa & $\begin{array}{l}26 \text { sesiones tutoría: } \\
1 \text { semanal } \\
\text { 54EF: } 2 \text { semanales }\end{array}$ & Curso 2012-13 \\
\hline \multirow{2}{*}{ Observación } & $\begin{array}{c}\text { Obser vación participante de las } \\
\text { actitudes y relaciones que se } \\
\text { dieron entre el alumnado }\end{array}$ & Diario abierto & Curso 2012-13 \\
\hline & $\begin{array}{l}\text { Comparación de las mejoras en } \\
\text { la resolución de conflictos } \\
\text { respecto a los resultados iniciales }\end{array}$ & $\begin{array}{c}\text { Observación sistemática de } 22 \\
\text { sesiones de EF }\end{array}$ & 3r trim. 2012-13 \\
\hline \multirow{2}{*}{ Reflexión } & Análisis de datos obtenidos & $\begin{array}{l}\text { Análisis cualitativo: Nvivo } \\
\text { Análisis cuantitativo: SSPS }\end{array}$ & 3r trim. 2012-13 \\
\hline & $\begin{array}{l}\text { Reflexión sobre los resultados y } \\
\text { propuestas de fututo y de mejora }\end{array}$ & Grupo de discusión/ Entrevistas & 1r trim. 2013-14 \\
\hline
\end{tabular}

clima de convivencia de los docentes y los alumnos, sentando las bases para una intervención posterior. A su vez, permitió establecer una priorización de las CCBB a tratar y los objetivos, contenidos y criterios de evaluación que guiarían su consecución. En la tabla 2 encontramos las dimensiones de la CSC, en la tabla 3 las dimensiones del resto de competencias tratadas, y en la tabla 4 los objetivos y contenidos pautados de manera interdisciplinar.

Una vez presentadas las CCBB a conseguir y su concreción en los objetivos y los contenidos planteados, vamos a dar paso a explicar la metodología didáctica desarrollada para lograrlo.

Puesto que se partió de un enfoque competencial, los métodos de enseñanza aplicados buscaron facilitar el pensamiento crítico y el desarrollo de habilidades de comunicación útiles para desenvolverse en múltiples entornos (Monjas, 2008; Ruiz Omeñaca, 2008). Para ello, fue necenecesidades de la investigación, puesto que a partir de una comisión formada por docentes del centro interesados en el tema, se elaboró el plan de acción, se llevó a cabo una reflexión constante de la práctica y se estructuró una rigurosa recogida de datos a lo largo de todo el ciclo. De este modo, a lo largo del proceso todos los maestros de la comisión colaboraron en la toma de decisiones y aprendieron progresivamente a través de sus experiencias. En la tabla 1 mostramos un resumen de las acciones llevadas a cabo en cada fase de la I-A y las herramientas empleadas para la recogida de datos.

Una vez situados en la I-A vamos a dar paso a presentar el programa de acción estableciendo las estrategias de planificación, la metodología y un conjunto de tareas a desatacar por su Tabla 2 .

Dimensiones de la CSC tratadas (basado en Ruiz Omeñaca, J. V.; Ponce de Leon, A.; Sanz, E. \& Valdemoros, M.A., 2013)

\section{Dimensiones CSC}

-SCS.1. Ex perimentar y conocer la diversidad de perspectivas de análisis de la sociedad. -SCS.2. Valorar como una riqueza las aportaciones de la diversidad cultural.

-SCS.3. Entender las características del proceso democrático ejerciendo el derecho de participar en la toma de decisiones como miembro de un grupo.

-SCS.4. Construir, aceptar y practicar normas de convivencia.

-SCS.5. Crear a partir de la reflexión crítica y el diálogo una escala de valores de acuerdo a los patrones culturales básicos.

-SCS.6. Ser consciente de los valores del entorno y evaluarlos.

-SCS.7. Dominar habilidades sociales para resolver autónomamente los conflictos, inherentes en la convivencia, constructivamente mediante el diálogo y la negociación.

-SCS.8. Adoptar una actitud respetuosa y de cooperación.

-SCS.8. Adoptar una actitud respetuosa y de cooperación.

ideas para controlar la conducta.

-SCS.10. Desarrollar la sensibilidad y la creatividad para afrontar los conflictos.

perspectiva innovadora.

\section{Programa de acción}

Dada la problemática en el clima de convivencia que percibían los docentes, en primer lugar se decidió reflejar objetivamente dicha realidad. Para ello se emplearon dos estrategias: la observación sistemática de las sesiones de EF, del tercer trimestre del curso 2011-12, para determinar el número, causas y resoluciones de los conflictos dados; y un cuestionario para el alumnado, que partía de las aportaciones de Ruiz Omeñaca (2008) y Capllonch (2008), para averiguar la percepción del alumnado sobre el clima de convivencia y el nivel de conflictividad en el aula y en EF. Estas dos herramientas permitieron elaborar un informe de diagnóstico de la situación inicial y establecer mayores niveles de congruencia entre la visión del

Tabla 4.

Objetivos y contenidos del programa de acción

Objetivos

Aumentar la participación e implicación activa en la mejora de la convivencia del centro.

Desarrol lar las capacidades de reflexión hacia los conflictos que se dan en la escuela y optar

por la op ción más acorde con los valores tratados: respeto, igualdad, tolerancia y solidaridad, Utilizar los es pacios y materiales según las normas.

Desarrol lar la capacidad para dial ogar, las habilidades sociales y las actitudes neces arias para afrontar constructivamente las relaciones de convivencia.

Desarrol lar la respons abilidad adquirien do estrategias de autorregulación y autocontrol para

RC y evitar la aparición de conductas violentas.

Valorar el rendimiento y eléxito sin que el resultado influya en el disfrute y las relaciones interpersonales.

Fomentar las habilidades sociales neces arias para trabajar en grupos cooperativos: reflexión, participación, diálogo, autorregulación y tolerancia.

Desarrol lar sentimientos de empatía hacia los compañeros.

Expresar emociones y sentimient os mediante el gesto y el movimiento.

saria la combinación de diferentes estilos de aprendizaje que partían de la realidad del alumno y buscaban su experimentación a través de propuestas globales, abiertas y de colaboración que requerían motivación y capacidad para aprender de otros y

\begin{tabular}{|c|c|}
\hline CCBB & Dimensiones \\
\hline $\begin{array}{l}\text { Comunicativo } \\
\text { lingüística }\end{array}$ & $\begin{array}{l}\text {-CL1. Exp resar adecua damente pensamientos, emociones, vivencias y opiniones. } \\
\text {-CL2. Desarrollar la asertividad, empatía, actitudes de diálogo y RC. } \\
\text {-CL3. Utilizar la argumentación para encontrar diferentes sol uciones a un } \\
\text { problema. } \\
\text {-CL4. Aceptar y realizar críticas con espíritu constructivo. } \\
\text {-CL5. Adaptar la propia comunicación a los requisitos de la situación. }\end{array}$ \\
\hline $\begin{array}{l}\text { Conoci } \\
\text { mundo }\end{array}$ & $\begin{array}{l}\text { - CI1. Interpretar el mun do aplicando conceptos y principios para analizar } \\
\text { situaciones desde diferentes perspectivas. }\end{array}$ \\
\hline $\begin{array}{l}\text { Artística y } \\
\text { cultural }\end{array}$ & $\begin{array}{l}\text {-AC1. Reelabo rar formas de transmitir ideas y sentimientos propios y ajenos. } \\
\text {-AC2. Desarrollar el sentido de pertenencia a una cultura determinada y el } \\
\text { respeto hacia otras formas culturales diferentes a la propia. }\end{array}$ \\
\hline $\begin{array}{l}\text { Aprender a } \\
\text { aprender }\end{array}$ & $\begin{array}{l}\text {-AA1. Ser capaz de autoevaluarse y autorregularse. } \\
\text {-AA2. Mostrar respons abilidad y compromiso personal. } \\
\text {-AA3. Saber admin istra r el esfue zzo, a ceptar los errores y apren der de y con los } \\
\text { demás. } \\
\text {-AA4. Respetar y colaborar ac tivamente y mostrar disposición para ayudar a } \\
\text { otros en diferentes situaciones de apren dizaje. } \\
\text {-AA5. Fomentar habilidades sociales como el trabajo en equipo, cooperación, } \\
\text { reparto de tareas, implicación y participación, y protocolos y técnicas a seguir en } \\
\text { la RC. }\end{array}$ \\
\hline $\begin{array}{l}\text { Iniciativa } \\
\text { personal }\end{array}$ & $\begin{array}{l}\text {-IP1. Desarrollar un autoconcepto crítico en el que se reflexione sobre los } \\
\text { valores, actitudes y motivaciones. } \\
\text {-IP2. Autocontrol emocional para vivir en paz con uno mismoy poder responder } \\
\text { de manera acorde a la diversidad de situaciones y contextos de relación } \\
\text { interpersonal. }\end{array}$ \\
\hline
\end{tabular}

para generar conocimiento (Pérez Gómez, 2007).

La aplicación del programa se desarrolló semanalmente en una sesión de tutoría y dos de EF de una hora cada una. Los espacios empleados fueron el patio y la sala de psicomotricidad para EF; y el aula del grupo clase, el patio y la sala de informática para las sesiones de tutoría. Gracias a la disponibilidad de espacios, en las sesiones de tutoría se pudo distribuir al alumnado en dos subgrupos para preparar ciertas actividades o cambiar de espacio cuando las tareas lo requerían.

Cada sesión de tutoría se centraba en un contenido que la mayoría de las veces se trataba con un estilo de enseñanza expositivo dialogal, el maestro exponía el tema a través de preguntas que los alumnos mismos respondían organizando sus conocimientos o experiencias. Una vez expuesta la tarea se daba paso al método de redescubrimiento por equipos (Quevedo \& González Ruiz, 2012), de modo que cada grupo trabajaba un 
aspecto y elaboraba unas conclusiones que compartía con el resto de la clase en los minutos finales de la sesión. Estas reflexiones solían plasmarse en murales y carteles, que posteriormente se colgaban en el aula como recordatorio. Las herramientas de reflexión solían ser lluvias de ideas, el razonamiento moral a través de historias hipotéticas (Ruiz Omeñaca, 2004), resolución de problemas o debates en torno a valores. Estas estrategias de reflexión estaban enfocadas a combinar conocimientos, habilidades y actitudes para resolver problemas, próximos a la realidad del niño, de manera activa, autónoma y responsable (Blázquez, 2009).

Una vez tratadas las capacidades y habilidades relacionadas con la CSC en las sesiones de tutoría, se continuaban desarrollando en unidades didácticas de EF, relacionadas con los cinco bloques de contenidos del área (Decret 142/2007; Real Decreto 1513/2006), que las potenciaban gracias a la metodología activa y cooperativa establecida. Las sesiones de EF seguían la estructura del modelo Hellison (1995): presentación inicial de los objetivos en valores o conductas a seguir en clase, formulados a partir de una pregunta que los alumnos debían tener presente en toda la sesión para autoevaluarse al finalizarla, propuesta de actividades motrices en relación a diferentes contenidos con posibles interrupciones para reflexiones necesarias, y reflexión final para evaluar la sesión y autoevaluar las conductas individuales. Desde este punto de vista el docente era un guía que estimulaba, acompañaba, implicaba al alumno y evaluaba para ir llevando a cabo las modificaciones necesarias para mejorar los procesos (Pérez Gómez, 2007).

El trabajo en equipo era esencial para conseguir los objetivos de las sesiones de las dos áreas, conllevando diálogo, debate, respeto a las diferencias, saber escuchar, enriquecerse con las aportaciones ajenas y tener la generosidad suficiente para ofrecer lo mejor de sí mismo (Zabala, 2011). Además, en muchos momentos debían responsabilizarse de aquello que aprendía el compañero, por lo que las habilidades comunicativas continuaban siendo claves para ofrecer críticas constructivas, observaciones, correcciones y reflexiones. Al trabajar en pequeños grupos, también se generó un grupo afectivamente más accesible que permitía que todos se integraran, aceptaran sus diferencias, se incrementaran las dinámicas de ayudas mutuas y se favoreciera la creación de lazos de amistad (Velázquez Callado, 2013).

Una vez establecida la metodología didáctica, consideramos que interesante explicitar cuatro actuaciones de éxito destacadas por el alumnado:

\section{Creación de normas}

Durante el primer año de vida del centro no existieron unas normas claras de convivencia, ni para el alumnado, ni para el profesorado, por lo que en algunas ocasiones se dieron situaciones que dificultaron el funcionamiento colectivo del centro. Dada esta realidad y considerando que las normas son necesarias para el respeto mutuo y la regulación de la convivencia (Prat \& Soler, 2003), la primera acción del programa, realizada durante el curso 2012-13, fue la de crear un normativa de aula a través de un proceso democrático. En la elaboración de las normas los protagonistas fueron los estudiantes, ya que se partió de la premisa de que para la formación de personas implicadas en la sociedad es necesaria la práctica de la ciudadanía (Marina \& Bernabeu, 2007). Una vez propuestas las normas en una asamblea de clase, se transformó su redacción en enunciados positivos para la buena convivencia. Después el alumnado decidió generar una serie de consecuencias para las personas que las transgredieran y por último se aprobó cada norma con una votación unánime. Ellos mismos clasificaron las normas en cuatro ámbitos: saber estar en el aula, respeto a los compañeros, el patio y EF. Paralelamente en EF se llevó a cabo una unidad didáctica (UD, de aquí en adelante) llamada Creando juegos, dónde el objetivo principal era concienciar al alumnado de la importancia del cumplimiento de las normas para el buen funcionamiento de los juegos y la escuela. De estas asambleas también surgieron ideas para gestionar y administrar el día a día como fueron los cargos mensuales (lista, orden, repartidor, material de EF y plástica, biblioteca) o el diario de la clase como medio para la regulación de las relaciones personales y de la convivencia. De este modo se fomentó la responsabilidad del alumnado ante sus actos, la distribución de los cargos y la reflexión.

\section{El grupo como protagonista}

También queremos destacar las actividades generales del centro como estrategia para la implicación del alumnado en el clima de convivencia. En las festividades en las que participaba todo el alumnado del centro, los alumnos de quinto y sexto llevaban el peso de las tareas relacionadas con la organización (presentación, repartición de material, preparación de la decoración, acompañamiento a los más pequeños de la escuela u organización de juegos y danzas). Estas actividades buscaban potenciar el sentimiento de pertenencia e identidad al grupo para mejorar la autoestima colectiva (Marina \& Bernabeu, 2007).

\section{El banco de tiempo escolar}

El banco de tiempo escolar (BdTE, de aquí en adelante) (Pujol \& Cacho, 2009) fue otra de las actuaciones de éxito llevadas a cabo. Después de tratar diferentes habilidades sociales para la mejora de las relaciones interpersonales y la autoestima, se buscó potenciar las capacidades individuales de cada alumno a través del BdTE, aplicado quincenalmente en las sesiones de tutoría y puntualmente en las de EF para sistematizar el aprendizaje entre iguales. En la primera fase se propusieron unas actividades previas que ayudaban a decidir que cuatro habilidades, de cualquier ámbito, se querían enseñar. Una vez fijadas las ofertas de cada alumno, los alumnos agrupados por parejas pactaban los contenidos que se enseñarían y firmaban un contrato de ayuda mutua. En la sesión siguiente debían estar preparados para enseñar durante 20 minutos su habilidad y aprender la del compañero durante los siguientes 20 minutos. Después del intercambio se evaluaban tanto como docentes como dicentes y, a continuación, se daba paso a formar las parejas de la siguiente sesión y a firmar el nuevo contrato (Pujol \& Cacho, 2009). Con el BdTE los alumnos aprendieron a ofrecer y recibir ayudas de un compañero, se pusieron en práctica las habilidades para el diálogo trabajadas y se continuó reforzando la autoestima. En este punto, cabe resaltar que el trabajo en parejas hizo surgir explicaciones, de los contenidos planteados, más cercanas y comprensibles para los puntos de vista de los alumnos (Quevedo \& González Ruiz, 2012). En cuanto a las habilidades que se enseñaron, destacaron las relacionadas con contenidos de EF y educación visual y plástica. El BdTE también se implementó en EF. Para ello se destinaron las últimas sesiones de cada trimestre mediante grupos de trabajo de cuatro personas en las tareas relacionadas con las habilidades motrices.

\section{El contrato de paz}

La última acción que vamos a describir es el contrato de paz, que solo se aplicó en el caso de alumnos que tenían problemas de control de la agresividad, tanto física como verbal, en el momento de lidiar con los conflictos o en sus interacciones con los compañeros. Si un alumno mostraba más de cinco actitudes de este tipo a lo largo de dos semanas, desde la asamblea de la clase se podrían proponer la elaboración de un contrato de paz. Después de una charla individual con la tutora, el alumno debía firmar un documento para comprometerse a mejorar su actitud hacia los compañeros y controlar sus impulsos. El contrato de paz llevaba adjunto un cuadernillo, donde el niño o niña debía anotar aquellas situaciones en que sentía que iba a perder el 
control y lo que sucedía después, tanto si pudo solucionarlo positivamente, como negativamente. Cada quince días el alumno y la maestra se volvían a reunir para valorar la evolución y considerar si se debía continuar con el trabajo de reflexión. El objetivo del contrato de paz era hacer consciente al alumno de las situaciones que le alteraban y de como las gestionaba.

\section{Evaluación}

Paralelamente a la fase de acción de la I-A, también se realizó la de observación para detectar a tiempo los puntos débiles del proceso de enseñanza y de investigación, y así reconducir lo planificado (Latorre, 2003). Según Zabala (2011), evaluar competencias es evaluar procesos de resolución de problemas contextualizados, complejos y productivos; en situaciones reales o recreadas en el aula, para que el alumno aplique de forma activa sus conocimientos. Desde esta perspectiva, la evaluación competencial facilitó una visión integradora del proceso de aprendizaje y permitió comprobar si existía transferencia de conocimientos entre contextos y autonomía del aprendizaje. Así pues, la evaluación que nos planteamos permitió contrastar el grado de consecución de los objetivos didácticos fijados y regular el proceso de aprendizaje, al permitir adaptar las estrategias pedagógicas a las características del alumnado y a sus progresos (Pellicer \& Ortega Delgado, 2009). La evaluación también se consideró como un elemento de aprendizaje, por lo que se establecieron estrategias de autoevaluación y coevaluación que involucraron al alumno en el proceso (Pérez Gómez, 2007). Desde este punto de vista la evaluación incrementó la motivación, autonomía y autoestima del alumnado, y le ayudó a entender sus dificultades y a encontrar caminos para mejorar (Comisión Europea, 2012).

A continuación presentamos los instrumentos que permitieron recoger datos para valorar si el alumnado adquiría las habilidades de la CSC y detectar los posibles cambios en el clima de convivencia. Como ya hemos comentado anteriormente, una observación sistemática y un cuestionario para el alumnado permitieron obtener la información necesaria para la evaluación inicial. A lo largo de todo el programa de acción se llevó a cabo una evaluación a través de observaciones participantes en las sesiones de EF y tutoría. Esto permitió evaluar al alumnado sin que la presencia del observador modificara su conducta. Estas observaciones se recopilaron en un diario abierto del docente (Latorre, 2003), en el que se describían los hechos observados y los resultados, y se reflexionaba sobre el funcionamiento de las tareas propuestas y sobre las actitudes y relaciones que se dieron entre el alumnado. Como herramienta de coevaluación se propuso el diario de clase, donde cada alumno podía plantear dudas, felicitar a compañeros, presentar quejas, expresar deseos, opiniones o reflexiones; para que se pusieran en común en las asambleas de clase. Por otro lado, en cada sesión de EF se recurría a la autoevaluación de la habilidad social central del día y en cada UD de EF el maestro evaluaba los objetivos motrices y sociales planteados. Como última herramienta para recoger datos individuales del alumnado, se recurrió a sus producciones: reflexiones escritas u orales, murales o acciones motrices. Éstas permitieron enfrentar a los estudiantes a lo que habían comprendido y al docente le ayudaron a constatar su nivel de dominio y aplicación. La evaluación llevada a cabo al final del programa permitió valorar los logros conseguidos, aplicando las habilidades trabajadas en diferentes situaciones y contextos. Para ello, de nuevo se recurrió a la observación sistemática de 22 sesiones de EF del tercer trimestre del curso 2012-13 con la colaboración de un observador entrenado. Esta observación nos sirvió para comparar los resultados del inicio del proceso con los del final, ya que se observaron las mismas categorías pre-establecidas. Por otro lado, dentro de la fase de re- flexión de I-A se llevaron a cabo entrevistas y grupos de discusión con maestros implicados en el proyecto, para recoger su percepción sobre las mejoras conseguidas, pero dada su extensión no se recogen en este artículo.

\section{Resultados}

Para el análisis estadístico de los resultados cuantitativos obtenidos a través del cuestionario administrado en la fase de planificación y la observación sistemática se empleó el software PASW Stadistics 18 (SSPS) y para los datos cualitativos de las actas, los diarios y los grupos de discusión, el software Nvivo 1.1

Sobre los datos obtenidos en el cuestionario, podemos afirmar que al menos un 68,9\% de los alumnos manifestó que a veces se sentía mal en la escuela, aunque percibían que en las sesiones de EF se daban igual o menor número de conflictos que en el aula. Por otro lado, pudo detectarse que para los alumnos las principales causas de los conflictos dados en EF eran las faltas de respeto $(23,8 \%)$, los problemas generados en la formación de grupos $(19,4 \%)$ y las diferencias entre los niveles de habilidad (12,9\%). En cambio, como podemos ver en el siguiente párrafo extraído del diario del maestro, el docente percibía otros hechos como origen de los conflictos:

En el día a día de las sesiones de EF siento que los alumnos tienen muchos problemas a la hora de aceptar los resultados y jugar limpiamente. Las peleas más fuertes aparecen cuando alumnos que ejercen un rol de líder negativo sobre el grupo, están perdiendo y pierden el control mostrando agre-

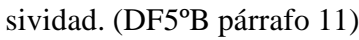

Los resultados relacionados con la percepción que tenían los alumnos de sus reacciones ante los conflictos siguieron una línea similar a la anterior variable analizada. Mientras que los alumnos mayoritariamente consideraron que cuando tenían un conflicto respondían ignorándolo (27,7\%), se lo decían al maestro para que ayudara a solucionarlo $(22,2 \%)$ o bien hablaban con la otra persona $(21,1 \%)$; desde el equipo docente se sentía que las principales actuaciones eran insultar, faltar al respeto o incluso agredir físicamente, o ignorarlo si la otra persona era un líder del grupo. Así lo podemos entrever en este fragmento del acta de la segunda reunión de la comisión docente:

En el centro docente tenemos un serio problema, nuestros

alumnos no saben controlar se cuando tienen un problema y sus impulsos le llevan al insulto y al golpe para imponer su razón, necesitan ser capaces de dialogar y buscar soluciones para las dos partes. (ACD3 26-09-12)

Las observaciones participantes de EF, tanto del inicio del proceso como del final, revelaron una realidad distinta a la percepción de los alumnos. Al principio los insultos (22,8\%), el dejar de participar (14\%) y las discusiones (14\%) eran las respuestas inmediatas y al finalizar la intervención si que fueron más reales, puesto que predominaron el ignorar el hecho (24,5\%), el diálogo (24,5\%) o el pedir ayuda a un compañero o al maestro $(9,4 \%)$.

Dado este cambio en las reacciones inmediatas al conflicto, podemos afirmar que mejoraron las dinámicas para solucionar los conflictos positivamente. Mientras que antes de la aplicación del programa se detectaron un 4\% de agresiones físicas, al finalizarlo no existió ninguna. La disminución del número de contratos de paz también fue un indicador de la mejora de la convivencia. En el primer trimestre se firmaron seis, en el segundo tres y en el tercero ninguno. Otro cambio substancial, fue el incremento de la aparición de habilidades sociales relacionadas con el diálogo y la ayuda entre iguales.

Durante la sesión de hoy se han dado unos cuantos accidentes, debido a que el patio estaba un poco mojado. Para mi ha sido increíble ver como ellos solos ofrecían ayuda a los 
compañeros que se habían hecho daño y se preocupaban porque estuviera bien. Al inicio de curso era inimaginable, cuando alguien se caía llegaban hasta a reírse. (DF3, párrafo 72)

Hoy Juan se ha enfadado un poco porque decía que no le tocaba parar y Miguel decía que sí. Ha estado muy bien ver como los dos mantenían el control, daban su opinión y se escuchaban. Ellos solos han decidido repetir el tiro, porque no les quedaba demasiado claro. (DF3, párrafo 81)

Esta semana no me he peleado con nadie, me he sabido controlar y cuando Federico me ha dado una pata jugando a futbol, me he acordado que él es un poco bruto y le he dicho que tuviera cuidado muy tranquilamente, sin enfadarme y él me ha preguntado si estaba bien. Creo que nos lo pasamos mejor en el cole si no nos peleamos. (DC, párrafo 29)

Estos resultados nos permite considerar que el programa planificado ha dado sus frutos, ya que se han introducido mejoras en diversas dimensiones de la CSC, como son: los procesos de RC, las ayudas mutuas, la no transgresión de las normas, el autocontrol, y la implicación en la mejora de la convivencia.

\section{Discusión y conclusiones}

El buen clima de convivencia de un centro educativo es esencial para crear contextos donde el alumnos pueda aprender y disfrutar de ello (Pareja y Pedrosa, 2012). Por lo tanto, intervenciones educativas dirigidas en esta línea serán beneficiosas para la mejora de la CSC, pero también del resto de CCBB (Hernández y Rodríguez, 2011). En cuanto a los resultados obtenidos al inicio del programa, se pone de manifiesto que alumnos y profesores no interpretaban de la misma manera la convivencia del centro, ya que la percepción de los estudiantes fue más optimista en relación a la variable de reacciones ante el conflicto. Si entendemos que el clima de convivencia es un factor importante en el proceso de enseñanza-aprendizaje, las divergencias encontradas en la forma de captar y entender la realidad cotidiana por parte de unos y otros podrían ser una razón de los problemas que se dan a diario (Ortega et al., 2008).

Trabajando interdisciplinarmente la tutoría y la EF se potenció el aprendizaje funcional y la creación de situaciones motivadoras que modificaban los contextos, fomentando la cooperación y aplicando como herramienta fundamental el juego. Así pues, desde la tutoría el alumnado reflexionó sobre cómo se relacionaba con los compañeros y cómo ello afectaba a la convivencia; $\mathrm{y}$ en el entorno de EF experimentó nuevas maneras de relacionarse y de afrontar los conflictos. Esta metodología competencial perseguía aplicar los conocimientos adquiridos en un contexto diferente y más activo y espontáneo como es el de la EF (Ruiz Omeñaca, 2008). Así también se minimizaron posibles dinámicas negativas relacionadas con la competitividad que pueden surgir en EF (Velázquez Callado, 2013) y se aprovecharon sus características para ayudar a aprender a convivir, a respetar la autonomía personal, la participación y la apreciación de la diversidad (González et al., 2011). A su vez, el trabajo cooperativo aplicado favoreció desarrollar habilidades fundamentales como la comunicación oral, el análisis o el trabajo en equipo (Velázquez Callado, 2013) y el aprendizaje del respeto y la aceptación de las reglas en los juegos y actividades deportivas como medio para entender la importancia que tiene el actuar según las normas para mejorar la convivencia (Lleixà, 2007).

Por otra parte, aplicando esta intervención se consiguió concienciar al profesorado sobre la problemática existente y se demostró que sí era posible el cambio con un trabajo reflexivo y elaborado por parte de maestros y alumnos. En definitiva, estaba en las manos de todos el conseguir crear un clima más acoge- dor para aprender y desarrollarse (Gómez, Latorre, Sánchez y Flecha, 2006).

\section{Referencias}

Blázquez, D. (2009). Las competencias básicas. En D. Blázquez y E. M. Sebastiani (Eds.), Enseñar por competencias en Educación Física (pp. 63-81). Barcelona: Inde.

Bolívar, A. \& Moya, J. (2007). Las competencia básicas. Cultura imprescindible de la ciudadanía. Proyecto Atlántida.

Capllonch, M. (2008). Juega, dialoga y resuelve: la superación de conflictos en E.F. mediante el modelo comunitario. Diseño de un programa especifico para Comunidades de aprendizaje. Proyecto con referencia SEJ2007-61757/EDU. Plan Nacional I+D+I. Universidad de Barcelona. Centro especial de investigación en teorías y prácticas superadoras de desigualdades. Disponible en http://utopiadream.info/ca/centros-en-funcionamiento/experiencias-en-ca/educacion-fisicaen-ca/

Casey, A., Dyson, B. \& Campbell, A. (2009). Action research in physical education: focusing beyond myself through cooperative learning. Educational Action Research, 17 (3), 407-423.

Cerezo, F. (2007). La violencia escolar. Propuestas para la intervención eficaz. Comunicación presentada en el Observatorio de la convivencia escolar. Jornadas sobre conflictos y convivencia en los centros escolares, Murcia, 26 a 28 de abril de 2007. Disponible en http://www.jornadas c onvivenciamurcia.com/ponencias/ cerezo_ramirez_fuensanta-violencia_escolar _propuestas.pdf

Comisión Europea. (2012). Education and Training 2020. Work programme Thematic Working Group 'Assessment of Key Competences'. Literature review, Glossary and examples. Bruselas. Disponible en http://ec.europa.eu/education/ policy/school/doc/keyreview_en.pdf

Decret 142/2007, de 26 de juny, pel qual s'estableix l'ordenació dels ensenyaments de l'educació primària. Diari oficial de la Generalitat de Catalunya no 4915 de 29 de juny de 2007.

Doncel, J. \& Leena, M. (2011). Las competencias básicas en la enseñanza. Fundamentación, enseñanza y evaluación. Sevilla: Editorial MAD.

Escamilla, A. (2008). Las Competencias Básicas. Claves y propuestas para su desarrollo en los centros. Barcelona: Graó.

Felip, N. (2013). Els processos de millora en l'àmbit de la convivència a les escoles de primària de Catalunya i les principals dificultats d'implementació. Pedagogia i Treball Social, 3(1), 67-86.

Fernández-Rio, J. (2003). El aprendizaje cooperativo en el aula de educación física par la integración en el medio social. Análisis comparativo con otros sistemas de enseñanza y aprendizaje. Valladolid: La Peonza.

Fernández-Río, J., \& Velázquez, C. (2005). Desafíos físicos cooperativos. Sevilla: Wanceulen.

Garrett, R. \& Wrench, A. (2011). Making Physical Education a Fairer, Safer and Happier Place: Putting Critical Practices into Action. Asia-Pacific Journal of Health, Sport and Physical Education, 2(2), 35-49.

Gázquez, J.J., Pérez, M.C. \& Carrión, J.J. (2011). Clima escolar y resolución de conflictos según el alumnado: un estudio europeo. Revista de Psicodidáctica, 16(1), 39-58.

Gómez, I. \& Prat, M. (2009). Hacia una Educación Física y transformadora de las prácticas sociales: de la práctica a la refelxión y viceversa. Cultura y Educación, 21 (1), 9-17.

Gómez, J., Latorre, A., Sánchez, M. \& Flecha, R. (2006). Metodología comunicativa crítica. Barcelona: El Roure. 
González, S., Catalán, M. \& Lara, J. M. (2011). La competencia social y ciudadana en Educación Física. En O. R. Contreras \& R. Cuevas (Eds.), Las competencias básicas desde Educación Física (pp. 63-77). Barcelona: INDE.

Hellison, D. R. (1995). Teaching personal and social responsability through physical activity. Champaign: Human Kinetics.

Hernández, J. M. \& Rodríguez, A. J. (2011). Contribuciones de la Educación Física al desarrollo de la competencia social y ciudadana. Acción Motriz, 7, 38-47.

Hine, G. \& Lavery, S. (2014). Action Research: Informing Professional Practice within Schools. Issues in Educational Research, 24(2), 162-173.

Latorre, A. (2003). La investigación-acción. Conocer y cambiar la práctica educativa. Barcelona: Graó.

Lleixà, T. (2007). Educación Física y competencias básicas: contribución del área a la adquisición de las competencias básicas del curiculo. Tándem didáctica para la Educación Física, 23, 31-37.

Marco Stiefel, B. (2008). Competencias básicas. Hacia un nuevo paradigma educativo. Madrid: Narcea.

Marina, J. A. \& Bernabeu, R. (2007). Competencia social y ciudadana. Madrid: Alianza.

Martín, M. \& Ríos, O. (2014). Prevención y resolución de conflictos en educación física desde la perspectiva del alumnado. Retos. Nuevas tendencias en Educación Física, Deporte y Recreación, 25, 162-167.

Monjas, M. I. (2008). Como promover la convivencia. Programa de asertividad y habilidades sociales (PAHS). Madrid: CEPE.

Moreno, M. A. (2012). Competencia social y ciudadana y coeducación. Gobierno de Navarra.

Moreno, D.; Estévez, E.; Murgui, S. \& Musitu, G. (2009). Relación entre el clima familiar y el clima escolar. Relación entre el clima familiar y el clima escolar: el rol de la empatía, la actitud hacia la autoridad y la conducta violenta en la adolescencia. International Journal of Psychology and Psychological Therapy, 9(1), 123-136.

Moreno-Murcia, J. A., Gónzalez-Cutre, D., \& Sicilia Camacho, Á. (2007). Metas sociales en las clases de educación física. Análisis y Modificación de conducta, 33(149), 5.

Norlena, S. \& Mohd, T. H. (2010). Facilitating the process of learning social skills though humanistic physical education. Procedia Social and Behavioral Sciences, 9, 223-227.

OCDE. (2007). Informe PISA 2006. Disponible en http:// www.bib.ufro.cl/portalv3/files/informe-pisa-2006.pdf.

Ortega, R., Del Rey, R., Córdova, F. \& Romera, E. M. (2008). Disciplina y gestión de la convivecia. 10 ideas clave. Barcelona: Graó.

Osman, G., Dilsad, M. \& Miiberra, C. (2010). Effects of cooperative game on social skill levels and attitudes toward physical education. Eurasian Journal of Eudcational Research, 40, 77-92.

Pareja, J. A. \& Pedrosa, B. (2012). Mejora de la convivencia a través de la investigación-acción participativa. Revista de Currículum y Formación de Profesorado, 16(3), 467-491.

Pascual, C., Escartí, A., Llopis, R., Marín, D. \& Wright, P. (2011). Implementation fidelity of a program designed to promote personal and social responsibility through physical education: A comparative case study. Research Quarterly for Exercise and Sport, 82(3), 499-511.

Pellicer, C. \& Ortega Delgado, M. (2009). La evaluación de las competencias básicas. Propuesta para evaluar el aprendizaje. Madrid: PPC.

Pérez Gómez, A. (2007). Las competencias básicas y el currículo. Gobierno de Cantabria. Consejeria de Educación.
Prat, M. \& Soler, S. (2003). Actitudes, valores y normas en Educación Física y el deporte. Barcelona: Inde.

Pujol, M. \& Cacho, X. (2009). Programa d'acció tutorial: banc del temps escolar. Perspectiva Escolar, 337, 57-61.

Quevedo, V. \& González Ruiz, D. (2012). Planificar y programar en los centros educativos. Departamentos didácticos y áreas de competencias. Madrid: Wolters Kluwer.

Real Decreto 1513/2006, de 7 de diciembre, por el que se establecen las enseñanzas mínimas de la Educación Primaria. BOE $n^{\circ}$ 293, de 8 de diciembre de 2006.

Real Decreto 1631/2006. de 29 de diciembre, por el que se establecen las enseñanzas mínimas correspondientes a la Educación Secundaria Obligatoria. BOE número 5 de 5 de enero de 2007.

Roberts, L., Yeomans, P. \& Ferro-Almeida, S. (2007). Project WIN. Evaluation shows decreased violence and improved conflict resolution skills for middle school students. Research in Middle Level Education, 30(8), 1-14.

Ruiz Omeñaca, J. V. (2004). Pedagogía de los valores en la Educación Física. Madrid: CCS.

Ruiz Omeñaca, J. V. (2008). Educación Física, valores éticos y resolución de conflictos: reflexiones y popuestas de acción. En A. Fraile (Ed.), La resolución de conflictos en y a través de la educación física. (pp. 65-116). Barcelona: Graó. Biblioteca Tándem.

Ruiz Omeñaca, J. V.; Ponce de Leon, A.; Sanz, E. 6 Valdemoros, M.A. (2013). La programación de educación física para primaria: propuesta para su elaboración. Logroño: Universidad de La Rioja.

Sánchez-Oliva, D., Leo, F. M., Amado, D., González-Ponce, I. \& López, J. M. (2012). Análisis diferencial de la percepción del desarrollo de valores en las clases de educación física. Retos. Nuevas tendencias en Educación Física, Deporte y Recreación, 22, 9-12.

Vaca, M. J. (2008). Contribución de la educación física escolar a las competencias básicas señaladas en la LOE para la educación primaria Tándem didáctica para la Educación Física, 26, 52-61.

Velázquez Callado, C. (2004). Las actividades físicas cooperativas. Una propuesta para la formación en valores a través de la educación física en las escuelas de educación básica. Materiales de actualización docente. Series: Educación Física. México: Secretaría de Educación Pública.

Velázquez Callado, C. (2008). Las actividades cooperativas como recurso para el tratamiento de los conflictos en las clases de educación física. In A. Fraile, V. M. López Pástor, J. Ruíz Omeñaca, \& C. Velázquez Callado (Eds.), La resolución de conflictos en y a través de la educación física (117162). Barcelona: Graó.

Velázquez Callado, C. (2013). Análisis de la implementación del aprendizaje cooperativo durante la escolarización obligatoria en el área de Educación Física. Valladolid: Servicio de Publicaciones de la Universidad de Valladolid. Disponible en https://www.educacion.gob.es/teseo/ mostrarSeleccion.do

Venero, J. P. (2007). La clase de Educación Física como motor de cambio social. Reflexionando sobre actividades en la naturaleza, curriculum oculto y valores. Retos. Nuevas tendencias en Educación Física, Deporte y Recreación, 11, 5153.

Zabala, A. (2011). Com cal ensenyar les competències i avaluar-les. En A. Zabala (Ed.), Què, quan i com ensenyar competències bàsiques a primària (pp. 29-48). Barcelona: Graó. 